\title{
The Ego Depletion and Its Relationship with the Future Anxiety among the University Female Students
}

\author{
Bassam AlHarbi ${ }^{1}$, Khaled Ibrahim ${ }^{2}$, Jaafar Al-Rababaah ${ }^{3} \&$ Salama Al- mehsin ${ }^{4}$ \\ ${ }^{1}$ Faculty of Educational Sciences, Department of Psychology and Psychological Counseling, Hashemite University, \\ Zarqa, Jordan \\ ${ }^{2}$ Assistant Professor of Educational Psychology College of Education, Prince Sattam bin Abdulaziz University, Al- \\ Kharj, Saudi Arabia \\ ${ }^{3}$ Professor of Educational Psychology, Psychology Department, Al-Balqa Aplied University, Amman, Jordan \\ ${ }^{4}$ Associate Professor Educational Psychology, The Ministry of Education, Amman, Jordan \\ Correspondence: Bassam AlHarbi, Faculty of Educational Sciences, Department of Psychology and Psychological \\ Counseling, Hashemite University, Zarqa, Jordan.
}

Received: October 12, 2020

Accepted: November 25, 2020

Online Published: November 30, 2020

doi:10.5430/ijhe.v10n2p128

URL: https://doi.org/10.5430/ijhe.v10n2p128

\begin{abstract}
The study aims to identify the degree of ego depletion and future anxiety and the relationship between both of them among the university female students. Researchers relied on the descriptive correlation method and prepared an ego depletion and future anxiety scale, and implement it on the study sample that consists of (560) female students at the Princess Alia University College after ensuring the appropriateness of their Seikometric characteristics. The study results found a statistically significant positive correlation between the ego depletion and future anxiety among the study sample, and the degree of ego depletion and future anxiety came medium. Results also showed that ego depletion acts as a good predictor of the future anxiety. In light of these results the study recommended the school administrations to provide students with the study environment that challenges their capabilities and provide them with the psychological safety to deal successfully with the future anxiety.
\end{abstract}

Keywords: future anxiety, ego depletion, university female students

\section{Introduction}

The future anxiety considers one of psychological problems that students suffer from at the present time, in light of the pressures they face due to fears of the future and the mysterious events it carries, which may not meet their ambitions, because the future anxiety takes a large part of their thinking (Al-Otaibi, 2016). The individuals' view of the future will be influenced by their personal characteristics and attributes, and in case these attributes are positive the future outlook will be consistent with the optimism and hope, but in the case of the negative attributes, the future outlook will be matched with pessimism and fear (Ju, Qin, Xu \& DiRenzo, 2016).

The personal characteristics also greatly affect the self-fulfilling or self-performing activities, such as the selection, decision-making, action control, control of desires, and self-regulation where all these activities require energy to perform and complete properly. This energy depends on limited sources within the body and can run out whenever the individual overstates or misuse these activities, which is known as the ego depletion. The Ego depletion is a low performance on a task that requires self-control, due to the effort made in previous tasks that required the activities of self-control, such as emotional control, behavior modification, and decision-making which lead to the poor self-control in performing the task (Friese, Loschelder, Gieseler, Frankenbach \& Inzlicht, 2019). The ego depletion also may represent the highest levels of psychological stresses and may affect the different relationships of individuals, which include the individual social relationships and the interactions with colleagues and family members (Maranges \& Baumeister, 2016).

\subsection{Ego Depletion}

A state of changeable decline in the ability of self-control that shows through feeling of psychological and physical attrition, poor performance of stressful tasks, distraction, and reduction in self-control ( $\mathrm{Li}$, Jiang, Yao \& Li, 2013). It's known procedurally; for the purposes of current study as the degree which respondent received on the ego depletion 
scale used in this study.

The psychological exhaustion or ego depletion is a temporary decline of energy required for self-control, which naturally consumed during the performance of its executive functions. (Li, Jiang, Yao \& Li, 2013) sees it as a state of psychological attrition and a lack of individual's internal energy that result from attempts to control and govern emerging conflicts between the inner desires of individuals and the reality that imposes a set of criteria and limitations on them.

The ego depletion can clearly affect an individual's performance, and there are many symptoms associated with the exhaustion. The first symptom is stress and tiredness that occurs as a result of the body's attempt to face effects of ego depletion; from the one hand and the chemical changes that occur to prevent the depletion on the other hand. Second symptom is poor performance (Alshaer \& Kaviani, 2019), third is difficulty of concentration, fourth is memory impairment (Derks, Van Mierlo \& Schmitz, 2014), and the fifth symptom is lack of control over motives (Bolton, Harvey, Grawitch \& Barber, 2012).

There are many theories that tried to explain the ego depletion and each theory tried to explain it within the limits of its vision, and here a presentation for some of these theories, the cognitive theory explains the ego depletion in light of the depletion of energy sources needed to perform cognitive processes, and its depletion return to the involvement of cognitive processes that require self-intervention to guide, control, and adjust the workflow of operations, which lead to a decrease in the performance level of processes (Alshaer \& Kaviani, 2019). The biological theory explained ego depletion, in term of "Amygdala Malfunction" which is a small area of the brain; near the center similar to an almond and responsible for the perception and organization of emotions by responding to the negative and positive incentives of emotions and feelings (Derks, Van Mierlo \& Schmitz, 2014). In the case of patients who suffer self-control disorders, this area responds more to the negative incentives ( $\mathrm{Li}$, Jiang, Yao, \& $\mathrm{Li}, 2013)$ and various disturbances or disorders occur, which eventually lead to the failure of self-control (Chen \& Li, 2020). The malfunction of "Amygdala" area caused by a reduction in the functional association between this area and other brain areas, such as the lateral frontal cortex and the central cortex which connected by the nerve fibers spread through the brain (Ju, Qin, Xu \& DiRenzo, 2016).

The owners of limited resources theory see that self-energy has a limited amount of resources used to perform many different tasks, such as self-regulation and thoughts, control of emotions, control of motives and desires, maintain of physical strength, and resistance to failure where these tasks consume these limited resources and therefore weaken the ability of individuals to organize and control themselves, which gradually leads to ego depletion (Bolton, Harvey, Grawitch \& Barber, 2012). Study results of (Ibrahim, 2017) indicated a significant reverse correlation between the ego depletion and psychological security. Other studies showed an interaction between self-efficacy and ego depletion like the study of (Al-Teh, 2013; Yaseen \& Ali, 2014).

Study results of (Thompson, et al., 2014) indicated that ego depletion reduces the ability to learn indirectly, while the study of (Al-Teh, 2013) found statistical significant differences between the ego depletion and self-efficacy. Study results of (Friese, Loschelder Gieseler, Frankenbach \& Inzlicht, 2019) indicated a statistically significant correlation between the ego depletion and depression while study results of (Sobocko, 2012) indicated that most sensitive individuals were more likely to develop ego depletion while consuming a large amount of energy, due to their involvement in negative emotions. (Darowski, 2011) found a negative and significant relationship between the ego depletion and self-regulation while (Friese, Loschelder Gieseler, Frankenbach \& Inzlicht, 2019) found statistically significant differences between males and females in the degree of ego depletion, in favor of males.

\subsection{Future Anxiety}

It is a feeling of tension and fear from the distant future, and its events that threaten the existence of individuals and prevent them from achieving their goals in one or more areas, such as personal, social, economic, occupational, and educational (Zaleski, 1996). It's defined procedurally for the current study purpose as the degree which the respondent received on the future anxiety scale used in this study. The concept of future anxiety shows through the general concept of anxiety as the perception of a state of apprehension, uncertainty, fear, panic, and doubt of undesirable changes in the personal future, and this will be the most tragic state of panic that happens to a person (Zaleski, 1996). It can also be defined as a negative temperament characterized by symptoms of physical stress and fear of the future, and it also characterized by a negative effect in term of the person concentration on the possibility of uncontrollable risk or misfortune (Lyu \& Huang, 2016).

Researchers define future anxiety as a feeling of uneasiness and a negative thinking towards the future, the negative perception of life, inability to cope with life pressures and events, low self-esteem, loss of feeling secure, and lack of 
self-confidence. The future anxiety means a state of tension, insecurity, and fear of unwanted changes in the future, and in its extreme state a threat that something will happen to the person who does not trust that future will bring him satisfaction and fulfillment, which will lead him to deactivate this future and stop the time towards the future.

The future anxiety is closely linked to pessimism, where a positive relationship found between the future anxiety and pessimism. (Alshaer \& Kaviani, 2019) confirmed that human who worry about the future characterized by pessimism, introversion, the appearance of doubt and hesitation signs, the appearance of emotions for the slightest reason and to fall under the influence of those emotions, and the tendency towards complexity, disorder, and instability. The person also has negative expectations of all that the future holds and the inability to face this future, where fear of the future leads to fear of the present and makes the person falls into a state of negativity.

The future anxiety is seen as extremism and exaggeration in caring for the future, which lead to a state of fear, tension, and uncertainty about the future, and the unwanted threats and changes that may occur in the future. Researchers define the future anxiety as a feeling of tension and fear from the distant future and its events that threaten the existence of individuals and prevent them from achieving their goals; in one or more areas. Freud considers one of the first to talk about the anxiety in psychology, where he assumed that origin of each anxiety is the trauma of childbirth, which represents the first experience of individuals with the anxiety and fear, and from this experience, patterns of reactions and feelings situations will be generated when individuals get exposed to a risk in the future, and can't overcome the anxiety that happen to them from this risk.

Pioneers of humanity theory believe that future anxiety arises when individuals can't give responses that lead to success in life situations and to satisfy social needs, which create conflicts among individuals and in turn lead to aggravate anxieties, particularly an anxiety from the future, and create feelings of dissatisfaction with the self (Alshaer \& Kaviani, 2019). However, Behaviorists view the future anxiety as something that human learn from the environment through acquired behaviors, especially mismatched behaviors that individuals learn in childhood, therefore it embedded in them and become characteristics of their personalities, which will be reinforced by the circumstances and environment that children live in it, and continue with them and cause them to not align with themselves and their environments which lead to anxiety.

While the authors of cognitive theory make connection between ideas and misperceptions and mental disorders, and they emphasize that these ideas can change behaviors through the intellectual reconstruction of patient's situations, which in turn strengthens the motivation for this change, therefore thoughts of individuals control their compatibility or incompatibility with themselves and their environment. Most researchers' interests focused on identifying the level of future anxiety among different samples of society members, where the university youth group had the largest portion such as the study of (Al-Sabawi, 2007; Zughebi, 2019; Al-Moumani \& Naeim, 2013; Al Qaisy \& Thawabieh, 2017), while other researchers turned toward schools' students such as (Al-Otaibi, 2016; Mohammed, 2014).

Study results of (Al-Sabawi, 2019; Zghebi, 2019; Moumani \& Naeim, 2013) indicated that future anxiety level among individuals was high, while study results of (Al Qaisy \& Thawabieh, 2017; Al-Lasasmeh, 2017) showed a moderate degree of future anxiety among individuals. There is also a relationship between the future anxiety and gender, in favor of females (Al-Sabawi, 2007) while study results of (Al Qaisy \& Thawabieh, 2017) indicated differences in the level of future anxiety and gender, in favor of males. The study of (Moumani \& Naeim, 2013) didn't find any differences in the level of future anxiety, due to gender and also didn't find any significant relationship between the future anxiety and academic specialization (Al-Sabawi, 2007; Zghebi, 2019).

The study of (Moumani \& Naeim, 2013) indicated no differences in the level of future anxiety, due to the academic level and it's possible to call for a negative and statistically significance relationship between future anxiety and the academic achievement, where every time the future anxiety increased the academic appreciation of students decreased (Al-Otaibi, 2016). Based on the above data and results, researchers noticed that previous studies emphasized the importance and newness of these two variables, and their powerful role on individuals' life and its relationships with many different psychological phenomena.

\section{Importance of the Problem}

The study gains its theoretical importance by highlighting one of the problems that experience by an important category in the society, which is university students and causes many negative aspects, such as the future anxiety as well as the ego depletion. These characteristics and features lead to waste of energies and cause frustration, which create many future negative effects on their lives, the attempt to determine its level, and its relationship with the future anxiety among this important group.

The current study will provide researchers and scholars with a theoretical framework of the future anxiety and ego 
depletion. One of the practical contributions of the current study that it helps staff in the psychological counseling units at the universities to understand the impact of these two variables on the life of students, which help to deal with them and predict their behaviors. The study also contributes to increasing community awareness that specialized in psychology of individuals who are under the control of future anxiety and ego depletion, which helps these individuals to seek help, and guide families toward helping their children to deal with future anxiety and ego depletion. In addition, the current study provides counselors and therapists with information about the spreading level of future anxiety and ego depletion in the community of university students, which helps them to take the necessary procedures and actions, and make the appropriate plans.

\section{Study Problems}

The university students face potential psychological risks and problems in a way that calls for the attention to do more researches on it, especially since they go through psychological, physical and social changes during the university study phase, which clearly contribute to the amplification of these problems, therefore it will affect their capabilities and potentials that may fade under these risks (Ju, Qin, Xu, \& DiRenzo, 2016).

In the shadow of this research approach, many studies confirm this direction that young people may suffer from anxiety in general and its symptoms appear on them. They may suffer from various forms of anxiety, such as future anxiety, intelligence anxiety, language anxiety, death anxiety, and performance anxiety, which in one way or another affects their capabilities, thoughts, ideas, and future aspirations (Fehm \& Schmidt, 2006; Kamarulzaman, Ibrahim, Yunus \& Ishak, 2013; Mirnics, Kov \& Bagdy, 2015; Al Qaisy \& Thawabieh, 2017; Yazid, Ali Khan, Asmuje, Zainun \& Theis, 2019).There are also the depression, violence, irrational thoughts, psychological stress, and the negative expectation of events (Kagan, Macleod \& Pote, 2004), the negative association with many of the positive aspects, such as efficiency and self-effectiveness (Al-Rasheidy, 2017; Al-LaSasmeh, 2017), the educational achievement (Al-Otaibi, 2016), and the motivation toward accomplishment, level of ambition, self-effectiveness, and self-esteem and appreciation (AlHarbi, 2014; Al-Mashieki, 2009; Blackhart, Williamson \& Nelson, 2015); Darowski, 2011; Sobocko, 2012). Therefore, the current study seeks to answer the following key question:

"What is the relationship between the ego depletion and future anxiety among the university female students?"

The following research questions stem from the key question above:

What is the degree of ego depletion among university female students?

What is the degree of future anxiety among university female students?

What is the relationship between the ego depletion and future anxiety among university female students?

What is the predictive ability of future anxiety among the university female students about their ego depletion?

\section{Aims and Hypotheses of the Study}

The current study aims to identify the following:

- The degree of ego depletion among the university female students.

- The degree of future anxiety among the university female students.

- The relationship between the ego depletion and future anxiety among the university female students.

- The predictive ability of future anxiety among the university female students about their ego depletion.

\section{Limitations of the Study}

The current study limited to the nature of its variables represented in the ego depletion and future anxiety, and the sample of 560 female students at the Princess Alia University College who registered during the first semester 2019/2020. The study also determined by its instruments and the interactivity level of sample members with it.

\section{Method}

\subsection{Study Procedures}

Researchers adopted the descriptive correlation approach due to its compatibility with the study nature, which aims to determine the degree of ego depletion and future anxiety and the relationship between them among female students of Princess Alia University College.

\subsection{Study Boundaries}

The current study limited to the nature of its variables represented in the ego depletion and future anxiety, and the sample of 560 female students at the Princess Alia University College who registered during the first semester 
2019/2020. The study also determined by its instruments and the interactivity level of sample members with it.

\subsection{Sampling Procedures}

The study sample consists of (560) female students in the bachelor phase at Princess Alia University College, where researchers used the categorical random method to select those regular students from the disciplines (Arabic Language, Child Education, Libraries, Special Education, Psychological Counseling, and English Language) for the academic year (2019/2020).

\subsection{Study Instruments}

The current study relied on the use of two tools, the ego depletion scale and the future anxiety scale and both prepared by researchers, as follow:

\subsubsection{First Ego Depletion Scale}

Researchers prepared the ego depletion scale in a way that align with the Jordanian environment; from one hand and in alignment with the current study sample and its psychological characteristics on the other hand, Researchers also reviewed a number of previous studies that have ego depletion scales in it, and in light of it the scale in its initial form consisted of (50) terms that reveal the ego depletion as a state of relative and changeable decline in self-control, which show through feeling of psychological and physical attrition, poor performance of stressful tasks, distraction, and reduced self-control.

\subsubsection{Validity of Scale}

The validity of instrument has been reached in several ways:

First: Arbitrators Validity

Researchers presented the scale on (10) arbitrators from faculty members at the psychology department but modified the language formulation of some items.

Second: Factorial Validity

Analyst calculated the factorial validity of scale by defining the factorial structure, where the factor analysis results for the exploratory sample degrees by Principal Component's method and the Orthogonal Rotation by Varimax's Method showed the explanation of four factors; in light of items' saturations which equal to $(0.35)$ or more. The first factor is a strong factor, where its "EIGNVALUE" after the Orthogonal Rotation amounted to (5.474) and it explained (11.90\%) from the overall variation ratio. This factor saturated with 14 items that revolve around things like the unwillingness to study, laziness and general weakness, inability to resist pressures, and inability to make decisions, therefore it called a feeling of attrition or feeling drained. The second factor is a strong factor, where its "EIGNVALUE" after the Orthogonal Rotation amounted to (4.517) and it explained (9.82\%) from the overall variation ratio. This factor saturated with 11 items that revolve around things like lack of motivation, and inability to accomplish difficult tasks, postpone it, and assign it to others, therefore it called poor performance. The third factor is a strong factor, where its "EIGNVALUE" after the Orthogonal Rotation amounted to (3.517) and it explained (7.65\%) from the overall variation ratio. This factor saturated with 11 items that revolve around things like the inability to concentrate and pay attention, feeling bored, and growing of forgetfulness, therefore it called distraction. The fourth factor is a strong factor, where its "EIGNVALUE" after the Orthogonal Rotation amounted to (2.627) and it explained (5.71\%) from the overall variation ratio. This factor saturated with 14 items that revolve around things like the inability to control emotions, weakness toward desires, and the inability to set priorities, therefore it called low self-control.

\subsubsection{Correlation Coefficient of Items with the Dimension and Overall Degree}

All Correlations Coefficient between each item and overall degree and the dimension that belongs to it were statistically significant, and the correlation coefficients between the items and overall degrees ranged from (0.39-0.79), while the correlation coefficients between the items and dimensions that belonged to range from (0.38-0.85), which are acceptable for current study's purposes.

\subsection{Second: Future Anxiety}

Researchers prepared the future anxiety scale in a way that align with the Jordanian environment; from one hand and in alignment with the current study sample and its psychological characteristics on the other hand, the scale in its initial form consists of (40) terms listed according to the Likert Fourth Scale, which reveals the future anxiety as a feeling of tension and a fear of the distant future, and its events that threaten the existence of individuals and prevent them from achieving their goals in the personal, social, economic, occupational, and academic fields. 


\section{Validity \& Reliability of Scale}

The scale implemented on an exploratory sample of (60) female students to verify its validity and reliability, through the following procedural steps:

Researchers verified the validity of scale to measure things intended for through the face validity, by presenting it on (10) arbitrators in psychology and deleted five phrases that didn't reach $80 \%$, and also analyst calculated the factorial validity of scale by defining the factorial structure, where the factor analysis results for the exploratory sample degrees by Principal Component's Method and the Orthogonal Rotation by Varimax's Method showed the explanation of five factors; in light of items' saturations which equal to $(0.35)$ or more. The first factor is a strong factor, where its "EIGNVALUE" after the Orthogonal Rotation amounted to (4.986) and it explained (10.74\%) from the overall variation ratio. This factor saturated with 8 items that revolve around things like pessimistic view of the future, expectation of inability to achieve goals, and ambiguity of the future, therefore it called the personal domain. The second factor is a strong factor, where its "EIGNVALUE" after the Orthogonal Rotation amounted to (4.712) and it explained $(9.29 \%)$ from the overall variation ratio. This factor saturated with 8 items that revolve around things like fear of changing society values, inability to establish successful social relationships, and fear of marital failure, therefore it called the social domain. The third factor is a strong factor, where its "EIGNVALUE" after the Orthogonal Rotation amounted to (4.280) and it explained (8.63\%) from the overall variation ratio. This factor saturated with 7 items that revolve around things like anxiety or apprehension about the increased burdens of life and living costs, and the inability of individuals to support them, therefore it called the economic domain. The fourth factor is a strong factor, where its "EIGNVALUE" after the Orthogonal Rotation amounted to (3.965) and it explained (5.93\%) from the overall variation ratio. This factor saturated with 8 items that revolve around things like work nature in the future, its relationship with education, and its compatibility with individuals' directions and tendencies, therefore it called the occupational domain. The fifth factor is a strong factor, where its "EIGNVALUE" after the Orthogonal Rotation amounted to (3.439) and it explained (5.93\%) from the overall variation ratio. This factor saturated with 9 items that revolve around things like possibility of achieving the academic excellence, fulfilling the expectations of parents and teachers, and the concern about exams, therefore it called the study domain.

\section{Scale Reliability}

Researchers confirmed the reliability of scale by using the Cornbach Alpha Coefficient and half-fragmentation or Splithalf coefficient. The reliability coefficient values using Cornbach Alpha coefficient ranged from (0.82-0.88) for dimensions and amounted to $(0.90)$ for the scale as a whole, but the reliability coefficient values using SpearmanBrown Split-half coefficient ranged from (0.85-0.91) for dimensions and amounted to (0.91) for the scale as a whole, which indicate that dimensions of the scale and the scale as a whole have high reliability.

\section{Results}

\section{Results of the first question:}

What is the degree of ego depletion among university female students? To answer this question, the analyst calculated arithmetic means and standard deviations of the ego depletion scale and its dimensions, and then compared it with the judgment criterion of medium to show the availability degree of each dimension. Results shown in the following table:

Table 1. arithmetic means and standard deviations for dimensions and overall degree of the ego depletion scale

\begin{tabular}{rccc}
\hline Dimensions & Means & STDEV & Availability \\
\hline Feeling drained & 3.06 & 2.55 & Medium \\
Poor performance & 3.20 & 2.71 & Medium \\
Distraction & 3.28 & 2.77 & Medium \\
Low self-control & 3.37 & 3.23 & Medium \\
Overall degree of ego & 3.22 & 5.80 & Medium \\
depletion scale & & &
\end{tabular}

Table 1 shows that ego depletion level among the study sample for dimensions as a whole came to a medium degree with an arithmetic mean of (3.22) and a standard deviation of (5.80).

\section{Results of the second question:}

What is the degree of future anxiety among university female students? To answer this question, the analyst calculated arithmetic means and standard deviations of the future anxiety scale and its dimensions, and then compared it with the 
judgment criterion of medium to show the availability degree of each dimension. Results shown in the following table:

Table 2. arithmetic means and standard deviations for dimensions and overall degree of the future anxiety scale

\begin{tabular}{lccc}
\hline Dimensions & Means & STDEV & Availability \\
\hline Personal & 3.14 & 2.46 & Medium \\
Social & 3.10 & 2.48 & Medium \\
Economical & 3.03 & 2.28 & Medium \\
Occupational & 3.35 & 2.31 & Medium \\
Academic & 3.18 & 2.74 & Medium
\end{tabular}

Table 2 shows that future anxiety level among the study sample came at medium degree, where its arithmetic means amounted to (3.17) with a standard deviation (5.58), also all dimensions came at medium degree

\section{Results of the third question:}

What is the relationship between ego depletion and future anxiety among university female students?

To answer this question, the analyst calculated the mutual correlation coefficients matrix between the overall degree and dimensions of both ego depletion and future anxiety, and results show in the following table:

Table 3 correlation coefficients matrix between the ego depletion and future anxiety among study sample

\begin{tabular}{|c|c|c|c|c|c|c|c|}
\hline Variable & Correlation/ Sig & Personal & Social & Economic & Occupational & Academic & $\begin{array}{l}\text { Overall Degree of } \\
\text { Future Anxiety }\end{array}$ \\
\hline \multirow[t]{2}{*}{ Feeling Drained } & Correlation Value & 0.150 & 0.094 & 0.072 & 0.123 & 0.131 & 0.119 \\
\hline & Sig & 0.943 & 0.875 & 0.653 & 0.984 & 0.769 & 0.857 \\
\hline \multirow{3}{*}{$\begin{array}{l}\text { Poor } \\
\text { Performance }\end{array}$} & Correlation & $0.680^{* *}$ & $0.745^{* *}$ & $0.658^{* *}$ & $0.543 * *$ & $0.624 * *$ & $0.751 * *$ \\
\hline & Value & 0.01 & 0.01 & 0.01 & 0.01 & 0.01 & 0.01 \\
\hline & Sig & & & & & & \\
\hline \multirow[t]{2}{*}{ Distraction } & Correlation Value & $0.593 * *$ & $0.781 * *$ & $0.637 * *$ & $0.601 * *$ & $0.563 * *$ & $0.739 * *$ \\
\hline & Sig & 0.01 & 0.01 & 0.01 & 0.01 & 0.01 & 0.01 \\
\hline \multirow{2}{*}{$\begin{array}{l}\text { Low } \\
\text { Control }\end{array}$} & Correlation Value & 0.143 & 0.078 & 0.093 & 0.069 & 0.176 & 0.165 \\
\hline & Sig & 0.876 & 0.760 & 0.765 & 0.783 & 0.657 & 0.743 \\
\hline \multirow{2}{*}{$\begin{array}{l}\text { Overall Degree } \\
\text { of Ego Depletion }\end{array}$} & Correlation Value & $0.451 * *$ & $0.563 * *$ & $0.521 * *$ & $0.497 * *$ & $0.511 * *$ & $0.664 * *$ \\
\hline & Sig & 0.01 & 0.01 & 0.01 & 0.01 & 0.01 & 0.01 \\
\hline
\end{tabular}

** Significant value at level 0.01

It shows from Table 3, on the overall degrees the existence of positive correlation between the overall degree of ego depletion and the overall degree of future anxiety, where the Pearson correlation coefficient value amounted to $(0.664)$ which is statistically significant at level $(0.01)$, while at the level of ego depletion dimensions, the correlation coefficient came positively correlated and statistically significant at level $(0.01)$ for the overall degree of future anxiety, in the dimensions of poor performance with a Pearson correlation coefficient value of $(0.751)$ and the distraction with a Pearson correlation coefficient value of (0.739).

The correlation coefficient came negatively correlated with the overall degree of future anxiety and not statistically significant; in the dimensions of feeling drained with a Pearson correlation coefficient value of (0.119) and low selfcontrol with a Pearson correlation coefficient value of (0.165).

\section{Results of the fourth question:}

What is the predictive ability of future anxiety among the university female students about their ego depletion?

To answer this question, the analyst calculated the multiple regression analysis by using the "Enter" method to identify the impact level of ego depletion's dimensions on the future anxiety to determine the dimensions that through it will 
be possible to predict the future anxiety among study members. Tables $4 \& 5$ explain the results of this analysis.

Table 4. Results of variation analysis, multiple correlation coefficient, and selection coefficient for the multiple regression model between the future anxiety and ego depletion's dimensions.

\begin{tabular}{cccccccc}
\hline Source & SS & DF & MS & F & Sig & R & $\mathrm{R}^{2}$ \\
\hline Regression & 774.71 & 4 & 193.68 & 5.166 & 0.001 & 0.286 & 0.082 \\
Error & 8697.39 & 552 & 37.49 & & & & \\
Total & 9472.10 & 556 & & & & & \\
\hline
\end{tabular}

Table 5. multiple regression coefficients and its statistical significance for the ego depletion's dimensions

\begin{tabular}{lccccc}
\hline \multicolumn{1}{c}{ Strategies } & $\mathrm{R}$ & Standard error & $\mathrm{SR}$ & $\mathrm{T}$ & Sig \\
\hline Fixed & 33.617 & 5.917 & & $5.681^{* * *}$ & \\
Feeling drained & 0.085 & 0.077 & 0.073 & 1.099 & 0.273 \\
Poor performance & -0.538 & 0.132 & -0.0386 & $-4.091^{* * *}$ & 0.000 \\
Distraction & 0.911 & 0.225 & 0.374 & $4.043^{* * *}$ & 0.000 \\
Low self-control & -0.045 & 0.052 & -0.055 & -0.866 & 0.387 \\
\hline
\end{tabular}

*** Significant value at level 0.001

It shows from Table 5 that multiple regression model between the future anxiety $(\mathrm{Y})$ and ego depletion's dimensions: feeling drained (X1), poor performance (X2), Distraction (X3), and low self-control (X4) can be formulated according to the following equation:

$$
Y=33.617+0.085 X 1-0.538 X 2+0.911 X 3-0.045 X 4
$$

This model indicates:

Fixed amount $=33,617$

Regression coefficients: $\mathrm{X} 1=0.085, \mathrm{X} 2=-0.0538$

$$
\mathrm{X} 3=0.911, \mathrm{X} 4=-0.045
$$

\section{The validity of estimated regression model:}

The validity of the estimated regression model can be judged by commenting on the multiple regression results of the ego depletion's dimensions shown in table (4) and table (5), where table (4) indicates that multiple correlation coefficient (R) is equal to 0.286 and that selection coefficient (R2) equal to 0.082 , which means that ego depletion's dimensions explain $8.2 \%$ from the changes that occur in the dependent variable (future anxiety), while the remainder $91.8 \%$ of the variation attributed to other factors. Therefore, the explanatory capability of the model considers inappropriate since it's less than $50 \%$ of the variation explanation of the study members' future anxiety. Table (4) which include variation analysis, also indicates that sig equal to 0.001 and its lower than the sig level of $1 \%$, therefore the regression model is statistically significant and will be possible to use the estimated regression model to make prediction about the future anxiety of study members.

It shows from table (5) which includes multiple regression coefficients and its statistical significances, that these coefficients came different in terms of its significance or nonexistence of statistical significance; from one hand and in terms of the significance level; on the other hand. It can be explained that fixed value in the equation is equal to (33.617) and this value has a statistical significance at level 0.01 , and therefore the presence of this constant or fixed value in the prediction equation is necessary and essential. It also noticed that regression coefficients feeling drained (0.085) and low self-control (-0.045) are non-statistically significant, and this finding suggests that these two dimensions are improper and invalid for the prediction about future anxiety.

It's also noticed from table (5) that regression coefficients of poor performance $(-0.538)$ and distraction $(0.911)$ are statistically significant, and this result indicates that these two dimensions are valid to be used as future anxiety alerts.

\section{Discussion}

Discussion of the first question: results show that ego depletion level among the study sample came at medium degree. The result can be explained in light of the characteristics of female students, which represent manifestations of strength and in the same time it represents reasons of disorder. For example, female students are overly sensitive which means 
growing emotion in the different life situations that lead to more pressure and negatively affects the individual's behavior, and at the same time they can use it as a source of energy and enthusiasm (Alshaer \& Kaviani, 2019).

In this context, the study of (Sobocko, 2012) indicates a positive correlation between sensitivity and the ego depletion, and also female students characterized with a tendency to perfection, which means the tendency of individual to set high personal criteria that are difficult to accomplish and far-reaching. These high criteria for success and goals achievement can be useful if individuals have the abilities to reach, but it may cause many negative emotions, such as anxiety, stress, depression, and dissatisfaction of life if individuals couldn't achieve these goals, and this what study results of (Albano, 2011; Guignard, Jacquet, Lubart, 2012)

There are also many academic challenges that face these female students, such as setting higher academic goals for themselves, looking into failure in more negative way, trying to meet the expectations of their teachers, competing with their colleagues, and trying to bring satisfaction from those around them, which increase tasks that require selfcontrol. Accordingly, these challenges and tasks lead to ego depletion while capabilities and potentials enable them to control themselves and reduce the fatigue or depletion. The nature of age group (19-21), which calls the adolescence stage leads to an increase in interests and ambitions and there are attempts for self-affirmation in the different areas of life that affect the concentration and attention of adolescent. Desires and lusts also increase significantly in this stage, and it will be hard for adolescents to face and control life situations (Derks, Van Mierlo \& Schmitz, 2014).

Discussion of the second question: results show that future anxiety level among the study sample came to a medium degree. This result agrees with the findings of some previous studies, such as (Al Qaisy \& Thawabieh, 2017; Yazid et al., 2019; Al-Lasasmeh, 2017), where these studies indicated that study sample showed a medium level of anxiety, and may explain this result in light of the different ideas, interests, directions, and tendencies. Therefore, the girl may be surrounded with a case of rejection and accusation by her peers, which in turn leads to increase her anxiety, and on the other hand the girl tries to gain experience by opening up to those around her and benefiting from them, which leads to a sense of social security and the resistance to anxiety.

It's possible to say that increase the future anxiety in the occupational field among the study sample returned to the ambiguity that surrounds this aspect of female students, where the future occupation in our country characterized by lack of clarity and the difficulty to predict it, in light of many factors, like the gap between education and job. The female student may excel in her studies and specialization but still doesn't find a work opportunity in her field.

Furthermore, the economic instability in the world countries in general and in our country in particular causes the opacity of occupational future and its lack of clarity, change the needs of labor market frequently, increase the population, and the lack of occupational jobs which greatly affects the perception of female students about their future career.

Discussion of the third question: results show the existence of positive correlation between the overall degree of ego depletion and the overall degree of future anxiety, while at the level of ego depletion dimensions, the correlation coefficient came positively correlated and statistically significant for the overall degree of future anxiety.

The correlation coefficient came negatively correlated with the overall degree of future anxiety and not statistically significant; in the dimensions of feeling drained and low self-control. The results indicate that an increase in the ego depletion will lead to an increase in the future anxiety, and a reduction in the ego depletion will lead to a reduction in the future anxiety, and emphasizes the need for self-control and the control of desires, which in turn reduces the future anxiety. This finding agrees with the study results of (Englert, Bertrams \& Kagan, 2012) which indicated a positive correlation between the ego depletion and general anxiety, as well as the study of (Al-Rashiedi, 2017) which showed a negative correlation between the future anxiety and self-control. Results can be explained in light of the human theory of anxiety, where Rogers sees lack of self-realization as one of the most important reasons for anxiety, and this perception derives from his view to the components of personality, which according to him consists of two components, the organic entity and the self-concept, and each has its own needs that require satisfaction in order for individual to achieve the tendency of growth and self-realization (Ju, Qin, Xu \& DiRenzo, 2016). This relationship between lack of goals' achievement and the ego depletion from one hand and the anxiety on the other hand confirms this positive correlation between the ego depletion and future anxiety. The achievement of objectives closely related to the strong willingness and internal energy that enables the individual to arrange his priorities, control his wishes, and give up his whims in order to achieve these goals. It also closely related to the psychological stability and feeling of psychological security that enables the individual to not surrender and continue the path until reaching the goal (Chen \& $\mathrm{Li}, 2020$ ).

This finding can be explained in light of the literary heritage of the ego depletion, where (Kaygusuz, Duyan, Oksal \& Duyan, 2015) referred to some aspects that confirm higher ego depletion among males than females, which include 
the prevalence of criminal and aggressive behaviors among males such as alcohol use, smoking, murders, violence, impulsiveness and recklessness, rebellion, failure to follow general laws and traditions and the attempt to break it, as well as the risk-taking behaviors such as driving at high speed and the violent games. (Kaygusuz, Duyan, Oksal \& Duyan, 2015) attributed that to several reasons, which include the poor control where males receive less control than females due to the social belief that male has the ability to take responsibility for himself more than the girl. In many situations, the education methods differentiate between male and female in rewards and punishments, where the punishment of girl may be more severe and more effective than the punishment of boy. As well as the nature of boy and his physical elements that may be a factor in obtaining everything he wants without control of this willingness, which leads in its entirety to a higher ego depletion among males than females. (Chen \& Li, 2020) The Study of (Al Qaisy \& Thawabieh, 2017; Al-Rashiedi, 2017) indicated an increase in anxiety levels among males more than females, and researchers explain this difference in light of the study sample differences in these studies, where the study sample represented in university students.

Discussion of the fourth question: results show that ego depletion acts as a good predictor of future anxiety, which means that poor performance and distraction are suitable for use as predictors of future anxiety.

Researchers attribute this result to the withdrawal of reality view on the future, which means a failure in the tasks of self-control related to the poor performance in difficult tasks, inability to complete these tasks, and the inability to solve problems, which involves the lack of desire and motivation to perform these tasks, and considers the first step to success and reaching the goal, as well as the distraction and lack of concentration which involve boredom and considers a strong barrier between the individual and success. All that have reflections on the individual's view of the future with some measurement of reality, where the more failure repeated in reality, the individual believes that failure will occur in the future as well, which increases his anxiety toward this future in a self-doubt about his abilities and potentials for success.

\section{Recommendations}

1) Activates the guidance services for female students within the university and society as a whole, which train female students on self-control to reduce the occurrence of psychological and social problems.

2) Offers training sessions on guidance and rehabilitation for female students to develop their self-confidence and benefit from their general capabilities and characteristics, which reduces their anxiety about the future.

3) Provides a school environment that challenges the capabilities of female students and gives them the psychological security about their academic future, which leads to no anxiety and no fear of the future.

\section{References}

Albano, A. (2011), "The Relationship among Perfectionism, Life Satisfaction, and Socio-Emotional Variables in Gifted Children", Doctoral dissertation, Hofstra University.

Al-Harbi, Tahani (2014), "The Future Anxiety and its relationship with self-esteem and the ambition level among high school female students in Riyadh", master thesis, Nayef Arab University for Security Sciences, Riyadh.

Al-Husseini, Atef Mesad (2011), "The Future Anxiety and the Treatment by Meaning", Arab Thought House, Cairo, Egypt.

Ali, Mohammed Al-Nubi (2017), "The Effectiveness of a Training Program based on Solving Future Problems to improve the Scientific Inference in reducing the Future Anxiety among mentally Excelling High School Students", Education Magazine, 172(2), 226-318, Al-Azhar University, Egypt.

Al-Lasasmeh, Mona (2017), "Self-Competence and Self-Management Methods and its Relationship with the Future Anxiety among Excelling High School Students at Al-Qasr Directorate of Education Schools", master thesis, Mu'tah University, Karak, Jordan.

Al-Mishekhi, Ghaleb Mohammed (2009), "The Future Anxiety and its Relationship with each of the Self-Effectiveness and Ambition Level among a sample of Taif University Students", Ph.D., Um Al-Qura University, KSA.

Al-Moumani, Mohammed Ahmed \& Naeim, Mazen Mahmoud (2013), "The Future Anxiety among Community College Students in Al-Galilee area, in light of some Variables", Jordanian Journal of Educational Sciences, 9(12), 173-185, Amman, Jordan.

Al-Otaibi, Hadhal Abdullah (2016), "The Impact of Future Anxiety on the Educational Achievement among a Sample of Secondary School Students", Journal of the Faculty of Education, 32(4), 426-457, University of Assiut, Egypt.

Al-Rashiedi, Benyan (2017), "The Future Anxiety and Self-Effectiveness among Students of Community College at 
Hail University, in light of some variables", Faculty of Education Journal, 174(2), 639-686, Al-Azhar University, Cairo, Egypt.

Al-Sabawi, Fadhilah (2007), "Studying the Future Anxiety among Students of the Faculty of Education, and its Relationship with Sex and Specialization", Faculty of Education, Mosul University, Iraq.

Alshaer, Hanouf \& Kaviani, Hossein (2019), "A study on metaphors used by female teachers to describe their workrelated stresses and Ego depletion, reflecting on potential interventions", Archives of Psychiatry \& Psychotherapy, Vol. 21, Issue 4, 72-81. https://doi.org/10.12740/APP/109135

Al-Teh, Ziad Khamies (2013), "The Relationship of Self-Effectiveness in Teaching with the Psychological Combustion and Experience among Teachers of Mafraq Governorate", Journal of the Union of Arab Universities for Education and Psychology, Syria, Vol. 11, No. 4, 163-189.

Al Qaisy, L. M., \& Thawabieh, A. M. (2017), "Personal Traits and Their Relationship with Future Anxiety and Achievement", Journal on Educational Psychology, 10(3), 11-19. https://doi.org/10.15640/jpbs.v4n2a11

Ameen, Osama Rabie (2008), “The Statistical Analysis using SPSS”, Anglo-Egyptian Library, Cairo, Egypt.

Blackhart, G. C., Williamson, J., \& Nelson, L. (2015), "Social anxiety in relation to self-control depletion following social interactions", Journal of Social and Clinical Psychology, 34(9), 747-773. https://doi.org/10.1521/jscp.2015.34.9.747

Bolton, L., Harvey, R, Grawitch, M., \& Barber, L. (2012), "Counter productive work behaviours in response to emotional exhaustion, a moderated mediational approach", Stress and Health, 28(3), 222-233. https://doi.org/10.1002/smi.1425

Burkley, E. (2008), "The role of self-control in resistance to persuasion", Personality and Social Psychology Bulletin, 34(3), 419-431. https://doi.org/10.1177/0146167207310458

Chen, Y., \& Li, S. (2020). Relationship between workplace ostracism and unsafe behaviors: The mediating effect of psychological detachment and emotional exhaustion. Psychological Reports, 123(2), 488-516. https://doi.org/10.1177/0033294118813892

Darowski, E. S. (2011), A Critical examination of the ego depletion effect, can you VS. Will you engage in effortful self- regulation? Doctoral dissertation, Michigan State University.

Derks, D., Van Mierlo, H., \& Schmitz, E. B. (2014), "A diary study on work-related smartphone use, psychological detachment and exhaustion: Examining the role of the perceived segmentation norm", Journal of Occupational Health Psychology, 19(1), 74-84. https://doi.org/10.1037/a0035076

Ego Depletion and its Relationship with the Depression among University Students, Seikometric-clinical study", master thesis, Faculty of Education, Ain Shams University, Egypt.

Englert, C., \& Bertrams, A. (2012), "Anxiety, ego depletion, and sports performance”, Journal of Sport and Exercise Psychology, 34(5), 580-599. https://doi.org/10.1123/jsep.34.5.580

Evans, J., \& Stanovich, K. (2013), "Dual-Process Theories of Higher Cognition, Advancing the Debate", Perspectives on Psychological Science, 8(3), 223-241. https://doi.org/10.1177/1745691612460685

Eyzenk, M., Payne S., \& Santos, R. (2006), "Anxiety and depression, past, present and future events”, Cognitive and Emotion, 20(2), 247-294. https://doi.org/10.1080/02699930500220066

Fehm, L., \& Schmidt, K. (2006), "Performance anxiety in gifted adolescent musicians", Journal of Anxiety Disorders, 20(1), 98-109. https://doi.org/10.1016/j.janxdis.2004.11.011

Friese, M., Loschelder, D., Gieseler, K., Frankenbach, J., \& Inzlicht, M. (2019), "Is ego depletion real? An analysis of arguments", Personality and Social Psychology Review, 23(2), 107-131. https://doi.org/10.1177/1088868318762183

Hamza, Jamal Mokhtar (2005), "The Future Anxiety among Offspring of Working Abroad", Journal of Educational Sciences, p.1, 89-11, Cairo University, Cairo, Egypt.

Ibrahim, Ryan Abdo (2017), "Psychological pressures and its relationship with the psychological security among couples who suffer from delayed reproduction, a field study at the fertility clinics and centers, and the treatment of delayed reproduction at the Khartoum State", master thesis, Faculty of Literature, African International University, Sudan. 
Guignard, J., Jacquet, A., \& Lubart, T. (2012), "Perfectionism and Anxiety: A Paradox in Intellectual Giftedness", PLOS ONE, 7(7), 1- 6. https://doi.org/10.1371/journal.pone.0041043

Ju, D., Qin, X., Xu, M., \& DiRenzo, M. (2016), "Boundary conditions of the emotional exhaustion-unsafe behavior link; the dark side of group norms and personal control", Asia Pacific Journal of Management, 33(1), 113-140. https://doi.org/10.1007/s10490-015-9455-7

Kamarulzaman, M. H., Ibrahim, N., Yunus, M. M., \& Ishak, N. M (2013), "Language Anxiety among Gifted Learners in Malaysia", English Language Teaching, 6(3), 20-29. https://doi.org/10.5539/elt.v6n3p20

Kaygusuz, C. Duyan, G. Oksal, A., \& Duyan, V. (2015), "The Effects of Some Socio-Demographic and Academic Characteristics of University Students on Self-Control”, American International Journal of Social Science, 4(5), 162-168.

Li, F., Jiang, L., Yao, X., \& Li, Y. (2013), "Job demands, job resources and safety outcomes, the roles of emotional exhaustion and safety compliance", Accident Analysis \& Prevention, 51, 243-251. https://doi.org/10.1016/j.aap.2012.11.029

Lyu, H., \& Huang, X. (2016), "Development and validation of future time perspective scale for adolescents and young adults", Time \& Society, 25(3), 533-551. https://doi.org/10.1177/0961463X16662669

Maranges, H. M., \& Baumeister, R. F. (2016), Self-control and ego depletion, handbook of self-regulation, Research, theory, and applications, 42-61.

Masood, Sana (2006), "Some Variables related to the Future Anxiety among a Sample of Adolescents, a Diagnostic Study", unpublished master thesis, Tanta University, Egypt.

Mirnics, Z., Kov, Z., \& Bagdy, E. (2015), Mental Health Promotion and Prevention among Gifted Adolescent, Cognitive-Counseling Research \& Conference Services Science, 3, 107- 120. https://doi.org/10.15405/book.3.2

Mohammed, Ghada Abdel Baki (2014), "A Proposed Perception to Reduce the Future Anxiety, from the standpoint of Students and Teachers at High School, Faculty of Education Magazine, 15, 604-627, Alexandria University, Egypt.

Sobocko, K. (2012), "The relationship between ego-depletion and sensory processing sensitivity", Doctoral dissertation, Carleton University. https://doi.org/10.22215/etd/2012-06777

Yaseen, Hamdi Mohammed \& Ali, Inas Sayed (2014), "Self-Effectiveness and Psychological Combustion among Teachers of Special Education", Faculty of Education Magazine, 25(97), 313-351. Binha University, Egypt.

Yazid A. F., Ali Khan A. R., Asmuje N. F., Zainun A., \& Theis N. A. (2019), "Correlation Study of Intelligence With Mental Health Issues Among Gifted and Talented Students", Malaysian Journal of Youth Studies, 2, 98-110.

Zaleski, Z. (1996), Future anxiety, concept, measurement, and preliminary research, personality and individual differences, 21(2), 165-174. https://doi.org/10.1016/0191-8869(96)00070-0

Zughebi, Mohammed Ahmed (2019), "The Ego Depletion among Students of University College at Haqel and its relationship with some variables, field study from the standpoint of University of Titbok Students", University College of Haqel, Higher Education Studies, Development Center for University Education, University of Assiut, Egypt.

\section{Copyrights}

Copyright for this article is retained by the author(s), with first publication rights granted to the journal.

This is an open-access article distributed under the terms and conditions of the Creative Commons Attribution license (http://creativecommons.org/licenses/by/4.0/). 